\title{
Predicting Trends in Social Networks via Dynamic Activeness Model
}

\author{
Shuyang Lin ${ }^{\dagger}$ Xiangnan Kong ${ }^{\dagger}$ Philip S. Yu ${ }^{\dagger *}$ \\ ${ }^{\dagger}$ Department of Computer Science \\ University of Illinois at Chicago \\ Illinois, USA \\ *Computer Science Department \\ King Abdulaziz University \\ Jeddah, Saudi Arabia \\ \{slin38,xkong4,psyu\}@uic.edu
}

\begin{abstract}
With the effect of word-of-the-mouth, trends in social networks are now playing a significant role in shaping people's lives. Predicting dynamic trends is an important problem with many useful applications. There are three dynamic characteristics of a trend that should be captured by a trend model: intensity, coverage and duration. However, existing approaches on the information diffusion are not capable of capturing these three characteristics. In this paper, we study the problem of predicting dynamic trends in social networks. We first define related concepts to quantify the dynamic characteristics of trends in social networks, and formalize the problem of trend prediction. We then propose a Dynamic Activeness (DA) model based on the novel concept of activeness, and design a trend prediction algorithm using the DA model. We examine the prediction algorithm on the DBLP network, and show that it is more accurate than state-of-the-art approaches.
\end{abstract}

\section{Categories and Subject Descriptors}

H.2.8 [Database Management]: Database ApplicationData Mining

\section{Keywords}

Information Diffusion; Social Influence

\section{INTRODUCTION}

Online social networks have become increasingly important for interpersonal communication and information sharing. Trends in online social networks now have large impacts on people's lives. Trends are represented by sequences of actions that are taken by users in a social network. According to the type of the social network, an action can be posting a blog or sharing a webpage about a certain topic, or joining an online activity.

Permission to make digital or hard copies of all or part of this work for personal or classroom use is granted without fee provided that copies are not made or distributed for profit or commercial advantage and that copies bear this notice and the full citation on the first page. Copyrights for components of this work owned by others than ACM must be honored. Abstracting with credit is permitted. To copy otherwise, or republish, to post on servers or to redistribute to lists, requires prior specific permission and/or a fee. Request permissions from permissions@acm.org.

CIKM'13, Oct. 27-Nov. 1, 2013, San Francisco, CA, USA

Copyright 2013 ACM 978-1-4503-2263-8/13/10 ...\$15.00.

http://dx.doi.org/10.1145/2505515.2505607.
Predicting the dynamic behavior of trends is an interesting problem with wide applications. Some examples of such applications are as follows:

1. Online video providers may want to predict how many times a video will be played by users in the next month, so that they can decide the bandwidth needed for the server. 2. Disease control facilities may want to predict how many people will suffer from a contagion in the following week, so that they can be prepared for an outbreak.

3. Manufacturers may want to predict how long an existing product will continue to be popular, so that they can decide the most suitable time for the debut of a new model.

The three applications above require the prediction of trends from three different perspectives. The first example considers the intensity of a trend, which is the volume of actions during a fixed length of time. The second one focuses on the coverage of a trend, which is the number of people taking the given action during a fixed length of time. The third one considers the duration of a trend, which is the time span that the intensity or coverage is above a given threshold.

To better explain these three perspectives (intensity, coverage and duration), we show in Figure 1 a toy example of a trend on a social network which contains three users. Table (b) shows the intensity, coverage and duration that aggregated from actions listed in Table (a). For example, at 2008, $v_{1}$ and $v_{2}$ take 3 and 2 actions, respectively, while $v_{3}$ taking no action, so the coverage (i.e. the number of people taking actions) is 2 , and the intensity (i.e., the total number of actions taken) is 5. Though the coverage and intensity are correlated with each other, they are not interchangeable in the sense that the corresponding time series are neither similar nor synchronized. In this example, the maximum value of coverage is reached at year 2009, while the maximum value of intensity is reached at year 2008. Duration reflects how long the trend lasts. If we set the threshold to 0, duration of the trend will be 4 years, from 2007 to 2010 .

Based on our observation, each of the three perspectives is useful for many real applications. A trend model should be able to characterize trends from all of these three perspectives.

Though the actions of social network users have been studied in the context of information diffusion models (e.g. the independent cascade (IC) model) [3], the existing information diffusion models are not suitable for modeling dynamic trends for three main reasons: First, most of these models assume that the diffusion processes take place in discretized time and the propagation of information between two nodes 


\begin{tabular}{llllll}
\hline & 2007 & 2008 & 2009 & 2010 & 2011 \\
\hline$v_{1}$ & 1 & 3 & 2 & 1 & 0 \\
$v_{2}$ & 0 & 2 & 1 & 0 & 0 \\
$v_{3}$ & 0 & 0 & 1 & 1 & 0 \\
\hline
\end{tabular}

(a) Number of actions of the trend

\begin{tabular}{llllll}
\hline & 2007 & 2008 & 2009 & 2010 & 2011 \\
\hline Coverage & 1 & 2 & 3 & 2 & 0 \\
Intensity & 1 & 5 & 4 & 2 & 0 \\
Duration & & \multicolumn{4}{c}{$2007-2010$}
\end{tabular}

(b) Coverage, intensity, and duration of the trend

Figure 1: An example of trend in a social network

always takes one unit of time, which does not reflect the real dynamic of trends as time unfolds. Therefore, they cannot reflect the dynamic nature of intensity and coverage, or the duration of trends. Second, information diffusion models focus on the visible path of propagation, and they usually assume that the propagation can only occur between a pair of nodes that are directly linked to each other, while the trend model should focus on predicting the aggregate characteristics of trends, and the path of propagation is not important for the prediction. Besides, because of the existence of homophily $[1,8]$, the propagation through direct links may not be good enough to explain trends in social networks. The model of trends should be more flexible with regard to the propagation mechanism. Third, information diffusion models focus on the prediction on the individual user level, but not on the trend level. As a result, they allow the probability of influence to be different between each pair of users, but assume that the probability remains the same for all the trends. This makes them not suitable for predicting trends based on different properties of trends.

In this paper, we formally define the three dynamic characteristics of a trend (intensity, coverage and duration), and the problem of trend prediction. We introduce a novel concept of activeness, which reflects a user's interest toward the given trend at a given point of time. The dynamic nature of activeness enables us to model the dynamic characteristics of trends. Due to the introduction of activeness, a more flexible propagation mechanism is made possible, so that the correlation as well as influence through direct links can be captured in our model. We propose a Dynamic Activeness (DA) model based on the concept of activeness. Each component of the model is built on observations on real trends, and the model is capable of capturing all the three dynamic characteristics of trends. We design a trend prediction algorithm based on the DA model. For each trend, the parameters of the model are learned specifically from the history data of that trend. The learned model can then be used to predict the dynamic characteristics of the trend in the future. We show the performance of the DA model on real trends in the DBLP network. A full version of the paper is available in [6].

\section{PRELIMINARIES}

\subsection{Notations and Definitions}

Let $G=(V, E)$ be a social network, in which $V=\left\{v_{1}, \cdots\right.$, $\left.v_{n}\right\}$ is the set of nodes and $E \subseteq V \times V$ is the set of edges. We consider the network to be static, since the evolution of networks is much slower than that of the trends. During the time span of a given trend, the change of the network is negligible.

A trend on the social network $G$ is defined as follows:

Definition 1. Trend $A$ trend $S=\left[\left(v_{1}, t_{1}\right), \ldots,\left(v_{m}, t_{m}\right)\right]$ on the social network $G$ is a chronological sequence that consists of a given type of actions in $G$, where $t_{i} \leq t_{j}(\forall 0 \leq i<$ $j \leq m)$ and $v_{i} \in V(\forall i \in\{1, \cdots m\})$. An element $\left(v_{i}, t_{i}\right)$ in $S$ corresponds to an action of that type taken by the node $v_{i}$ at time $t_{i}$.

For simplicity of notation, we denote the time sequence of actions in trend $S$ as $T(S)=\left[t_{1}, \ldots t_{m}\right]$, and denote the subsequence of $S$ that consists of all the actions taken by node $v$ as $S^{v}=\left[\left(v, t_{v, 1}\right), \ldots,\left(v, t_{v, m_{v}}\right)\right]$, where $\left(v, t_{v, i}\right) \in$ $S\left(\forall 1 \leq i \leq m_{v}\right)$. We also use $S_{t}$ to denote the prefix of $S$ which contains all the actions taken before the time point $t$, i.e., $S_{t}=\left[\left(v_{i}, t_{i}\right):\left(v_{i}, t_{i}\right) \in S, t_{i} \leq t\right]$.

Based on the above definition of a trend, we define intensity, coverage, and duration of a trend as follows:

DEFINITION 2. Intensity The Intensity of a trend $S$ on a time interval $I=\left[t_{\min }, t_{\max }\right)$ is the number of actions in $S$ that are taken during $I$. Formally, Intensity $(S, I)=$ $\left|\left\{\left(v_{i}, t_{i}\right): t_{i} \in I \wedge\left(v_{i}, t_{i}\right) \in S\right\}\right|$.

Definition 3. Coverage The Coverage of a trend $S$ on a time interval $I=\left[t_{\min }, t_{\max }\right)$ is the number of nodes in $\mathrm{V}$ that take at least one action during I. Formally, Coverage $(S$, $I)=\left|\left\{v_{i}:\left(v_{i}, t_{i}\right) \in S \wedge t_{i} \in I\right\}\right|$.

Definition 4. Duration Let $\mathcal{I}=\left\{I_{1}, \ldots I_{s}\right\}$ be a set of intervals, where $I_{i}=\left[t_{\text {min }}^{i}, t_{\text {max }}^{i}\right)$. Given a threshold $\theta$, the duration of $S$ on $\mathcal{I}$ is the number of consecutive intervals in $\mathcal{I}$ that the intensity (coverage) is above $\theta$. Formally, Duration $_{\text {cov }}(S, \mathcal{I}, \theta)=\max (j-i+1), 1 \leq i \leq j \leq$ s, s.t. $\forall k, i \leq k \leq j$, Coverage $\left(S, I_{k}\right)>\theta$ and Duration Dint $(S, \mathcal{I}, \theta)=\max (j-i+1), 1 \leq i \leq j \leq s$, s.t. $\forall k, i \leq k \leq$ $j, \operatorname{Intensity}\left(S, I_{k}\right)>\theta$.

The intensity quantifies the overall activeness of a trend within a social network. The coverage quantifies how broad a trend has impacts in a social network, i.e., the number of nodes involved within a time interval. The larger the coverage value of a trend is, the more nodes of the network are affected/involved in the trend. The duration quantifies how long a trend lasts within the social network.

For the duration, we usually want $I_{1}, \ldots, I_{s}$ to be consecutively connected intervals with equal length, i.e., $t_{\max }^{i}=$ $t_{\min }^{i+1}(\forall i \in\{1, \ldots, s-1\})$ and $t_{\max }^{i}-t_{\min }^{i}=t_{\max }^{j}-t_{\min }^{j}(\forall i, j \in$ $\{1, \ldots, s\})$. We like to point out that, given the intensity and coverage, the duration of trend can be defined in many different ways. We define it as the largest number of consecutive intervals above the threshold because this definition is most straightforward. By carefully setting the threshold $\theta$, the definition will accord with the intuitive understanding of the word "duration".

The prediction problem of trends is defined as follows:

Definition 5. Trend Prediction Problem Given $S_{t_{*}}$, the prefix of sequence $S$ before time $t_{*}$, the problem of trend prediction is to predict the intensity, coverage and duration of trend $S$ after time $t_{*}$.

Typically, we solve the prediction problem on a set of consecutively connected equal-length intervals $\mathcal{I}=\left\{I_{1}, \ldots I_{s}\right\}$, 
where $I_{i}=\left[t_{\text {min }}^{i}, t_{\text {max }}^{i}\right)$ and $t_{\text {min }}^{1}=t_{*}$. The problem is to predict Coverage $\left(S, I_{i}\right)$ and $\operatorname{Intensity}\left(S, I_{i}\right)$ for each $I_{i} \in$ $\mathcal{I}$, and Duration $\operatorname{cov}\left(S, \mathcal{I}, \theta_{S}\right)$ or Duration Dint $\left(S, \mathcal{I}, \theta_{S}\right)$ for a given $\theta_{S}$.

\subsection{Dataset}

We take our observation and evaluation on the DBLP coauthor network. In the network, the nodes correspond to the authors and the edges correspond to the co-authorship. The dataset contained 934,672 nodes and 8,850,502 edges. The trend data are extracted from the DBLP data by detecting terms in the titles of publications. In each trend, an action $\left(v_{i}, t_{i}\right)$ corresponds to a publication of the author $v_{i}$ at time $t_{i}$ that contains the given term in the title. For publications with multiple authors, there is an action for each of the authors.

\section{DYNAMIC ACTIVENESS (DA) MODEL}

\subsection{Concept of Activeness}

The DA model for trend in social network is based on the novel concept of activeness. For each trend, each node in the social network has an activeness function associated with it. The two main aspects of the concept are:

- Activeness of a node is defined as its interest toward the given trend. It is a function of time. As time goes by, activeness may increase as a result of information diffusion or social influence, or decrease as the node loses interest to the given trend.

- Activeness decides the frequency of actions taken by the node. The higher the activeness of a node is, the more actions it is likely to make in a unit time. In this sense, we can also define activeness as the "action rate" of a user.

Since activeness is dynamic in nature, we are able to design the DA model based on it, so that the model can capture the three dynamic characteristics of trends. Besides, by using activeness in the model, we are also able to design a more flexible information propagation mechanism.

\subsection{Framework of DA Model}

As shown in Figure 2, the DA model contains three elements: activeness propagation, decay of activeness and action generating process. Each of them is based on observations on real trends. Actions and activeness are connected to each other in the DA model. On the one hand, actions trigger activeness propagations in the social network. Activeness propagation, together with the decay of activeness, decides the activeness of each user at each point of time. On the other hand, actions are generated by the action generating process which takes the activeness as input.

As shown in Figure 2, the prediction algorithm contains two phases. In the learning phase, parameters of the DA model are learned from the observed part of trends. In the prediction phase, the DA model predicts the actions in the future. Intensity, coverage and duration of trends can be predicted by aggregating the predicted future actions.

\subsection{Activeness Modeling}

For each trend $S$, let $r_{v}(t)$ be the activeness or action rate of node $v$ at time $t$. In this section, we discuss the modeling of $r_{v}(t)$. As shown in the left most box in Figure 2, the model of activeness contains two parts: activeness propagation and activeness decay.

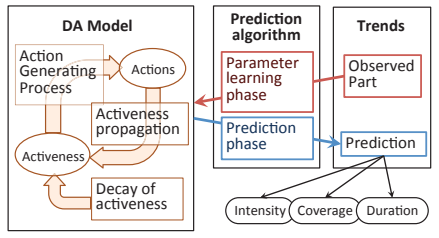

Figure 2: Block Diagram of the DA Model

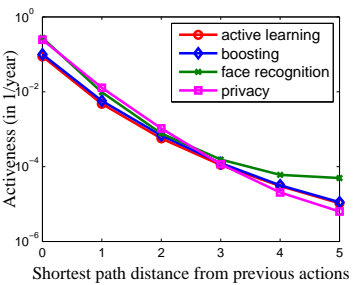

(a)

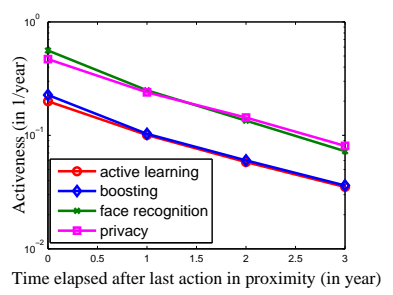

(b)
Figure 3: (a)Activeness by different distances to nodes with previous actions. (b)Activeness by the time elapsed since last action in proximity

Activeness Propagation Intuitively, as a result of social influence or homophily [8,2], the activeness of nodes are correlated with each other. The closer two nodes are, the larger the correlation is. Thus, when a node takes an action in a trend, we can expect that nodes in proximity to it have larger activeness for the trend than other nodes in the social network.

Our observation on the real trends supports this intuition. Figure 3(a) shows the curves of activeness for four trends in DBLP network, i.e., trends about "boosting", "privacy" etc. Other trends have similar curves. For each trend, we plot the average activeness (i.e. the average number of actions per unit time) for nodes with different shortest path distances to nodes with previous actions. As we can see from the figure, as the distance increases, the activeness of nodes exponentially decreases. An important remark is that the information diffusion along direct links is not enough for explaining trends. Because if we explain trends in that way, all the nodes except for those that are directly linked to the nodes with previous actions should have the same activeness. It is also interesting to point out the exponential decreases also fits for action rates of nodes with 0-hop distance (i.e. nodes themselves have previous actions).

Based on this observation, we use the previous actions of a trend to model the activeness of nodes. Let $\operatorname{prox}(u, v)$ be the proximity measurement from $u$ to $v$. When an action is taken by node $u$, the increase of activeness of $v$ is proportional to $\operatorname{prox}(u, v)$, i.e., if $u$ takes an action at time $t_{a}$, for every node $v$ in the network we have:

$$
\lim _{t \rightarrow t_{a}+} r_{v}(t)=\lim _{t \rightarrow t_{a}-} r_{v}(t)+\alpha \cdot \operatorname{prox}(u, v)
$$

where $\lim _{t \rightarrow t_{a}}+r_{v}(t)$ and $\lim _{t \rightarrow t_{a}-} r_{v}(t)$ are the activeness of node $v$ after and before the jump at time $t_{a}$, and $\alpha$ is the propagation ratio that depends on the trend.

We study two different measurements for proximity. The first one uses the shortest path distance from the source node to destination node. As we observed in the real trend data, proximity is defined as an exponential decreasing function 
of shortest distance, i.e., $\operatorname{prox}(u, v)=\exp (-b \cdot \operatorname{dist}(u, v))$, where $b \in \mathcal{R}^{+}$and $\operatorname{dist}(u, v)$ is the length of shortest path from $u$ to $v$. The second proximity measure is based on random walk, which is described as rooted PageRank in [5]. To measure the proximity of nodes from a given node $u$, the random walk is started at node $u$. At each step, it has a probability of $p$ to return to node $u$, and $1-p$ probability to move to neighbor nodes. The proximity of node $v$ from node $u$ is defined as the stationary probability for $v$. In both of the measurements, if $v$ is not reachable from $u$, we have $\operatorname{prox}(u, v)=0$.

While information diffusion models define the influence between nodes along the edges, the "propagation" of activeness captures a more general sense of correlation between activeness of nodes, rather than the process of information diffusion. Besides, it is different from information diffusion model in how it is parameterized. The parameter $\alpha$ of the activeness propagation depends only on the trend, but not on the node that takes the action. To the contrary, the information diffusion models usually have diffusion probabilities with individual edges as parameters, and the diffusion probabilities are constant for all the trends. We parameterize the activeness propagation in a different way for two reasons: First, it is simply not practical to make the parameters depend on trends and edges at the same time, since there will be not enough actions to be used for the learning of each parameter. Second, the main purpose of the proposed trend model is to predict the aggregate characteristics of different trends, so it is more meaningful to bind the parameter with the trends instead of the edges.

Decay of activeness Intuitively, if a user is not exposed to any new information or influence from a certain trend, nor does he create any new content that belongs to that trend, the user's interest to that trend will gradually decay. In other words, the activeness of a node spontaneously decays if there is no new action taken by nodes in proximity to it. This spontaneous decay is observed from real trends.

Figure 3(b) shows the average activeness for nodes as time progresses since last action in proximity. Let $R(v, k)=\{u \in$ $V \mid s p(u, v)=k\}$ represent the set of nodes that are $k$ hops away from $v$, where $s p(u, v)$ is the shortest path distance from $u$ to $v$. The $\mathrm{X}$-axis of the figure is the time elapsed since last action taken by nodes in $R(v, 1)$. The $\mathrm{Y}$-axis of the figure is the average activeness. As shown in the figure, activeness decreases when the node is not exposed to a new action. Decrease of activeness can roughly be regarded as exponential. (We only show the case of $k=1$ and the curves for four trends in this figure. But actually we have done the same test for different $k$ values and for different trends, and the other curves are similar.)

According to the observation, we introduce an exponential decrease to the activeness model. For each interval $\left[t_{0}, t_{1}\right)$ when $r_{v}(t)$ is not increased by the activeness propagation, $r_{v}(t)=r_{v}\left(t_{0}\right) e^{-\left(t-t_{0}\right) / \tau}$ for any $t \in\left[t_{0}, t_{1}\right) . \tau$ is a rate of the activeness decay, For similar reasons as to the parameter $\alpha$, $\tau$ depends on the trend, but not depends on the node or the edge.

Summary of Activeness Model Combining the two parts above together, $r_{v}(t)$, the activeness of node $v$ at time $t$, is given by:

$$
r_{v}(t)=\alpha \sum_{\left(v_{i}, t_{i}\right) \in S_{t}}\left(\operatorname{prox}\left(v_{i}, v\right) e^{-\left(t-t_{i}\right) / \tau}\right)+r_{v}\left(t_{0}\right) e^{-\left(t-t_{0}\right) / \tau}
$$

where $t_{0}$ is the start time of the trend. We set $r_{v}\left(t_{0}\right)$ to a small value $\epsilon . r_{v}(t)$ is discontinuous at time points when there is a new action taken by nodes in $R(v)$. In each interval between those discontinuous points, $r_{v}(t)$ is subject to an exponential decay.

\subsection{Action Generating Process}

As we mentioned in Section 3.1, the activeness of a node serves as the action rate in the generating process. Thus, we model the action generating process as a non-homogeneous Poisson process with the activeness as the rate parameter.

For a non-homogeneous Poisson process, the number of actions taken by this node in any time interval $\left[t^{\prime}, t\right)$ follows a Poisson distribution:

$$
P\left[\left(\left|S_{t}^{v}\right|-\left|S_{t^{\prime}}^{v}\right|\right)=k\right]=\frac{e^{-\int_{t^{\prime}}^{t} r_{v}(t) d t}\left(\int_{t^{\prime}}^{t} r_{v}(t) d t\right)^{k}}{k !}
$$

where $\left|S_{t}^{v}\right|$ is the number of actions taken by node $v$ before time point $t$.

Suppose we are now at the time point $t^{\prime}$ and want to generate the next time point after $t^{\prime}$. By taking derivative with respect to $t$, we can get the probability density function for the waiting time until next action:

$$
f_{v, t^{\prime}}(t)=r_{v}(t) \cdot \exp \left(\int_{t^{\prime}}^{t} r_{v}(t) d t\right)
$$

We then can generate the next time point in the sequence by drawing from the distribution of the waiting time.

\section{PREDICTION ALGORITHM}

The prediction algorithm contains two phases: the parameter learning phase and the prediction phase. In the first phase, parameters for each trend are learned from the part of the trend before $t_{*}$ by maximum likelihood estimation. In the second phase, we use the learned model to predict the future trend sequence after $t_{*}$. Efficiency implementation of the algorithm is available in the full version of this paper [6].

Parameter Learning Phase For each trend, two parameters in the DA model need to be learned: the proportionality factor $\alpha$ in activeness propagation and mean lifetime $\tau$ of activeness decay. We use maximum likelihood estimation for the parameter learning.

The likelihood function is given by:

$$
L(\alpha, \tau)=\prod_{v \in V} f\left(T\left(S_{t_{*}}^{v}\right),\left|S_{t_{*}}^{v}\right| ; \alpha, \tau\right)
$$

where $T\left(S_{t_{*}}^{v}\right)$ is the time sequence for node $v$ 's actions before time $t_{*}$, and $\left|S_{t_{*}}^{v}\right|$ is the number of actions taken by $v$ before time $t_{*} \cdot f(\cdot)$ is the joint probability density function of the time sequence $T\left(S_{t_{*}}^{v}\right)$ and $\left|S_{t_{*}}^{v}\right|$.

By taking the partial derivative of $\log L(\alpha, \tau)$ with respect to $\alpha$ (derivation is available in [6]), we get the estimate values for $\alpha$ :

$$
\hat{\alpha}=\frac{\left|S_{t_{*}}\right|}{\sum_{v \in V} H_{v}\left(t_{*}, \tau\right)}
$$


Fixing $\alpha$ to $\hat{\alpha}$, we get

$$
\begin{aligned}
\hat{\tau} & =\operatorname{argmax}\left[\left|S_{t_{*}}\right| \log \left(\frac{\left|S_{t_{*}}\right|}{\sum_{v \in V} H_{v}\left(t_{*}, \tau\right)}\right)-\left|S_{t_{*}}\right|\right. \\
& \left.+\sum_{\left(v_{i}, t_{i}\right) \in S_{t_{*}}} \log \left(h_{v_{i}}\left(t_{i}, \tau\right)\right)\right]
\end{aligned}
$$

where $h_{v}(t, \tau)$ and $H_{v}(t, \tau)$ are introduced for simplicity's sake:

$$
h_{v}(t, \tau)=\sum_{\left(v_{i}, t_{i}\right) \in S_{t}}\left(\operatorname{prox}\left(v_{i}, v\right) e^{-\left(t-t_{i}\right) / \tau}\right)
$$

and

$$
H_{v}(t, \tau)=\tau \sum_{\left(v_{i}, t_{i}\right) \in S_{t}}\left(\operatorname{prox}\left(v_{i}, v\right)\left(1-e^{-\left(t-t_{i}\right) / \tau}\right)\right)
$$

Due to the complexity of $H(t, \tau)$ and $h(t, \tau)$ with regards to $\tau$, it is not possible to obtain a closed-form solution for the maximum-likelihood estimate of $\tau$. However, since $\tau$ is the only variable here, we can use any line search algorithm to find the $\hat{\tau}$, and techniques such as simulated annealing can be adopted to avoid falling into local optimal value.

Prediction Phase After we learn the parameters $\tau$ and $\alpha$, we can generate the prediction of the action sequence after $t_{*}$. To do this, we keep track of the next action of each user in a list in the time order. Every time we pull the earliest action from the list and add it to prediction, then we update the list to capture the future actions that will be triggered by this action. The procedure is as follows:

1. Calculate $r_{v}\left(t_{*}\right)$, the activeness at time $t_{*}$ for each node $v$ in the network, using Equation 2.

2. Generate a next action for each node in the network from the pdf in Equation 3. Sort the actions in the ascending order of time, store them in a list $L$.

3 . While $L$ is not empty, pull the first action $\left(v_{i}, t_{i}\right)$ from it.

i if $t_{i}>t_{\text {end }}$, jump to step 4 .

ii Add $\left(v_{i}, t_{i}\right)$ to $S^{\prime}$, the predicted sequence.

iii For all the nodes that are reachable from $v_{i}$, update their activeness $r_{v}\left(t_{i}\right)$ by Equation 2 .

iv Update the next action time using the pdf in Equation 3, and sort the list $L$ again.

4. Calculate intensity, coverage, duration using the predicted sequence $S^{\prime}$.

$t_{\text {end }}$ in Step 3-i is the end point of the last time interval on which we want to predict the trend. Notice that the sequence generating process is a random process. We may repeat Step 3 several times to get the average value of aggregates.

\section{EXPERIMENT}

\subsection{Experiment settings}

Algorithms As we mentioned in Section 3.3, for the DA model, we use two different measurements for the proximity. For the shortest path measurement (DA-sp), we set the decay factor $b$ to 10 in the experiment. For the random walk measurement (DA-rw), the restart probability $p$ is set to 0.4 .

We compare the DA model with three variants of the widely used IC model. All of the three variants assume that each action comes with a delay, so that they can be used to model dynamic trends:

\begin{tabular}{|lll|}
\hline Concept drift & Boosting & Active learning \\
Kernel methods & Privacy & Streams \\
SQL & Face recognition & Decision tree \\
Heterogeneous network & & \\
\hline
\end{tabular}

Table 1: Trends in the DBLP dataset

1. eExp (Edge-dependent exponential delay model [7]) assumes that all the actions propagate through a certain edge are drawn independently from an exponential distribution. 2. tExp (Trend-dependent exponential delay model [7]) assumes that delays for all the actions of a certain trend are drawn independently from an exponential distribution.

3. tEqu (Trend-dependent equal-length delay model [4]) assumes that there is a fixed-length delay for all the actions of a certain trend.

Parameters of the three baselines model are learned by the algorithm proposed in [7]. For the intensity prediction, we extend the IC variants with a "multiple actions factor" to allow a node to perform actions more than once. These extended IC variants will be explained later in Section 5.3.

Performance Measures To evaluate coverage and intensity, we use two measures: the error ratio and the coefficient of variation. Error ratio is used to evaluate the goodness of the prediction compared with the true value. The formula of error ratio is given by error ratio $=\mid$ truth prediction $\mid /$ truth. Since all of the tested algorithms are stochastic algorithms, we also evaluate the variance of the outputs. For every test, we run each algorithm for multiple times and estimate the coefficient of variation. The coefficient of variation is estimated by: $\hat{C}_{v}=s / \bar{x}$ where $s$ is the sample standard deviation, and $\bar{x}$ is the sample mean.

Trend Data for Evaluation We test 10 trends of hot keywords in the areas of data mining and machine learning for the evaluation. Trends are as listed in Table 1. For each trend, we use the trend sequence before year 2005 (2005 excluded) as the training sequence, and the sequence from year 2005 to 2009 (2009 included) as the test sequence. We take each year as a time interval, on which we consider intensity and coverage.

\subsection{Coverage}

In Figure 4(a), we plot the average error ratio for 5 consecutive time intervals after the end point of training sequence. As shown in the figures, error ratios for all the algorithms tend to increase as time progresses. DA-rw and DA-sp have lower error ratios than the baselines. The error ratio of tEqu deteriorates very quickly, while eExp and tExp are in the middle. The difference between the error ratio of DA-rw and the error ratio of DA-sp is not large, which shows that the DA model is not sensitive to the different measurements of proximity, as long as the measurements are reasonable. In Figure 4(b), we show the coefficient of variation for the coverage prediction. As shown in the figure, DA-rw and DA-sp have a lower coefficient of variation than the baselines, which means that the predictions got by the DA model are more stable. DA-sp has a slightly lower coefficient of variation than DA-rw, which mean that the shortest path distance measurement makes the result more stable than the random walk measurement.

\subsection{Intensity}

For the evaluation of intensity prediction, we use tEqumult, tExp-mult and eExp-mult instead of tEqu, tExp, and 


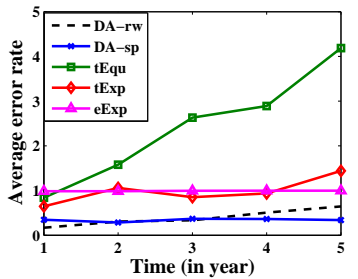

(a) Error Ratio

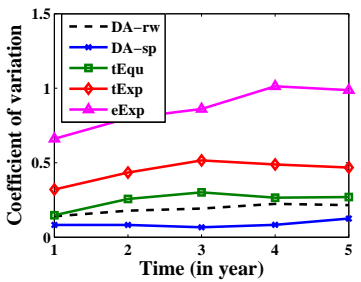

(b) Coefficient of Variation
Figure 4: Results for Coverage Prediction

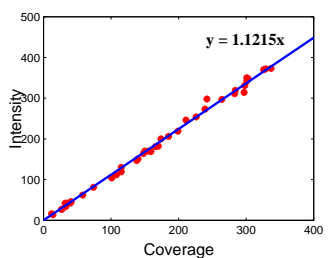

Figure 5: Relationship between Intensity and Coverage

eExp as baselines. It is because the tEqu, tExp and eExp, like the standard IC model, do not allow multiple actions being performed by the same node. To construct better baselines, we try to capture the relationship between coverage and intensity.

Figure 5 shows our observation on the relationship. We calculate coverage and intensity for all the intervals in the training time, and plot each pair of coverage and intensity in the figure. The values of coverage are illustrated on the $\mathrm{X}$-axes, and the values of intensity are illustrated on the $\mathrm{Y}$ axes. As shown in Figure 5, for this dataset, the proportional function $y=1.1215 x$ fits the relationship of intensity and coverage quite well, Based on this observation, we add a multiple action factor to the three baselines and get three new baselines.

Figure 6(a) shows the error ratios for intensity prediction. As shown in the figures, for all the algorithms, error ratios tend to increase as time progresses. DA-rw and DA-sp have lower error ratios than other algorithms. DA-rw performs slightly better than DA-sp. In Figure 6(b), we show the coefficient of variation for the intensity prediction. As shown in the figure, our algorithms have lower coefficient of variation than the baselines.

\subsection{Duration}

We test both coverage-based duration and intensity-based duration. To calculate the duration, we use the coverage and intensity at the last observed interval (year 2004) as thresholds. Since the length of the test time is limited, coverage or intensity for about half of the trends never drops below the thresholds. We make each algorithm predict whether the duration covers all the 5 prediction intervals, and report the accuracy of this prediction.

Table 2 shows the accuracy of the duration prediction. As shown in the table, DA-rw makes the best accuracy of prediction. The accuracy of DA-sp and tExp is similar. tEqu has the lowest accuracy.

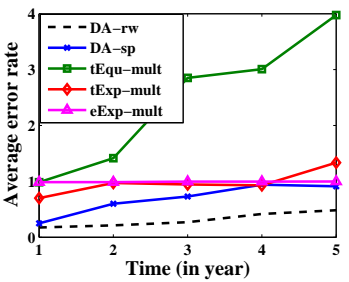

(a) Error Ratio

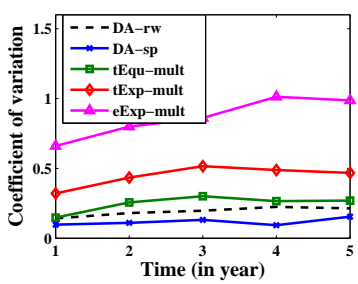

(b) Coefficient of Variation
Figure 6: Results for Intensity Prediction

\begin{tabular}{lcc}
\hline & Coverage-based duration & Intensity-based duration \\
\hline DA-rw & 0.9 & 0.9 \\
DA-sp & 0.8 & 0.9 \\
tEqu(-mult) & 0.6 & 0.5 \\
tExp(-mult) & 0.8 & 0.9 \\
eExp(-mult) & 0.7 & 0.7 \\
\hline
\end{tabular}

Table 2: Accuracy of Duration Prediction

\section{CONCLUSIONS}

In this paper, we identify coverage, intensity and duration as the three characteristics of a trend. We proposed a Dynamic Activeness model for trends based on the novel concept of node activeness. The model can capture all the three important aspects of trends. The experimental result shows that the proposed DA model can predict trends more accurately than information diffusion models.

\section{ACKNOWLEDGMENTS}

This work is supported in part by NSF through grants IIS-0905215, CNS-1115234, IIS-0914934, DBI-0960443, and OISE-1129076, and US Department of Army through grant W911NF-12-1-0066.

\section{REFERENCES}

[1] S. Aral, L. Muchnik, and A. Sundararajan. Distinguishing influence-based contagion from homophily-driven diffusion in dynamic networks. PNAS, 106(51):21544-21549, 2009.

[2] C. Budak, D. Agrawal, and A. El Abbadi. Structural trend analysis for online social networks. Proc. VLDB Endow., 4(10):646-656, 2011.

[3] D. Kempe and J. Kleinberg. Maximizing the spread of influence through a social network. In KDD, 2003.

[4] J. Leskovec, A. Krause, C. Guestrin, C. Faloutsos, J. Vanbriesen, and N. Glance. Cost-effective outbreak detection in Networks. In KDD, 2007.

[5] D. Liben-Nowell and J. Kleinberg. The link-prediction problem for social networks. Journal of the American Society for Information Science and Technology, 58(7):1019-1031, 2007.

[6] S. Lin, X. Kong, and P. S. Yu. Predicting trends in social networks via dynamic activeness model. CoRR, arXiv/1308.1995, 2013.

[7] K. Saito, M. Kimura, K. Ohara, and H. Motoda. Learning continuous-time information diffusion model for social behavioral data analysis. In $A C M L, 2009$.

[8] C. R. Shalizi and A. C. Thomas. Homophily and contagion are generically confounded in observational social network studies. Sociological Methods and Research, 40(2):211-239, 2011. 